Bio-grafía. Escritos sobre la Biología y su Enseñanza. ISSN 2027-1034

Número Extraordinario. p.p. 86-99

Memorias del Primer encuentro ambiental Universidad, ambiente y sustentabilidad: experiencias y prácticas.

\title{
LAS EXPRESIONES ARTÍSTICAS COMO PROPUESTA PEDAGÓGICA PARA FORTALECER LAS ACTITUDES AMBIENTALES FRENTE A LA QUEBRADA MORACÍ
}

\author{
Cortés Moreno Sindy Milena ${ }^{1}$ \\ Molina Andrade Nathalia ${ }^{2}$
}

\section{Resumen}

Esta investigación surge a partir de las diversas problemáticas ambientales halladas dentro de la Institución Educativa Campestre Monteverde, donde se evidenció la pertinencia de potenciar las actitudes pro-ambientales de los estudiantes frente a su entorno, observando que no poseen un reconocimiento amplio de su contexto, por lo cual no les es fácil proponer ideas que favorezcan la posible solución de dichas problemáticas. En esta medida, el propósito de este trabajo gira en torno al fortalecimiento de actitudes ambientales de los estudiantes frente a la Quebrada Morací a través de una estrategia pedagógica que incluya las expresiones artísticas. Para ello, se utilizó el enfoque cualitativo y el modelo de Investigación Acción, metodología que favoreció involucrar a los alumnos y reconocer con ellos las actitudes a fortalecer y las expresiones artísticas a trabajar. Así, se obtuvo que reconocen las problemáticas que afectan el contexto, determinaron como actitudes a fortalecer: el reconocimiento del ambiente, la proposición y resolución de problemáticas ambientales y la responsabilidad, con ayuda de expresiones artísticas como el dibujo, la literatura, el muralismo, entre otras.

\section{Palabras Clave}

Actitudes ambientales, expresiones artísticas, Quebrada Morací, estrategia pedagógica.

\footnotetext{
${ }^{1}$ Universidad Pedagógica Nacional. - Profesores en formación inicial - Departamento de Licenciatura en Biología - Línea de investigación: Educación en Ciencias y Formación Ambiental - dbi_scortesm483@ pedagogica.edu.co

${ }^{2}$ Universidad Pedagógica Nacional. - Profesores en formación inicial - Departamento de Licenciatura en Biología - Línea de investigación: Educación en Ciencias y Formación Ambiental - d d bi_nmolinaa556@pedagogica.edu.co
} 
Bio-grafía. Escritos sobre la Biología y su Enseñanza. ISSN 2027-1034

Número Extraordinario. p.p. 86-99

Memorias del Primer encuentro ambiental Universidad, ambiente y sustentabilidad: experiencias y prácticas.

\section{Abstract}

This investigation arises from the diverses environmental problematics found within educational institution Campestre Monteverde, where was identified the pertinence of strengthen environmental attitudes the students about their environment, observing that they do not recognize their context, so is not easy for them propose solution of these environmental problems. So, the purpose of this work is to strengthen the environmental attitudes of the students in front the Quebrada Moraci through a pedagogical strategy that includes the artistic expressions. So, was used the qualitative approach and model investigation-action, this methodology allowed to involve the students and recognize with they the attitudes to strengthen and artistics expressions to work. So, it's was found that they recognize the problematics that affect the context, they determined as attitudes to strengthen: the recognition of the environment, Proposing and solving environmental problematics, and the responsibility, with the help of autistics expressions such as drawing, Literature, and the muralism.

\section{Keys Words}

Environmental Attitudes, artistics expressions, Quebrada Morací, Pedagogical strategy.

\section{INTRODUCCIÓN}

El arte se ha convertido en un eje transversal para el enseñanzaaprendizaje de múltiples disciplinas, dado que ha permitido dinamizar e innovar los procesos académicos, por tanto, se han retomado para el desarrollo de esta investigación diversas expresiones artísticas planteando como objetivo principal fortalecer las actitudes ambientales en los estudiantes del grado 602 de la IED Campestre Monteverde el cual se encuentra en la localidad de Chapinero, de la cuidad de Bogotá.

Dicha investigación emerge a partir de un proceso de contextualización e indagación por parte de las maestras en formación dentro y fuera de la institución educativa; donde se encontró a nivel general que existe una marcada intervención antrópica de tipo negativo sobre el ambiente, el cual se caracteriza por poseer importantes ecosistemas acuáticos y terrestres; dentro de los cuales se localiza la Quebrada Morací, ecosistema acuático según (Hernandez, 2013) ubicado en los cerros orientales de la ciudad de Bogotá la cual posee diversidad de fauna y flora endémica de la zona, 
Bio-grafía. Escritos sobre la Biología y su Enseñanza. ISSN 2027-1034

Número Extraordinario. p.p. 86-99

Memorias del Primer encuentro ambiental Universidad, ambiente y sustentabilidad: experiencias y prácticas.

principalmente especies de colibríes, reptiles y anfibios, entre otros; hecho que se convierte en una importante posibilidad para abrir paso a futuras investigaciones de corte científico y académico. Además, es un cuerpo de agua de gran importante para la población, dado que, del nacimiento de esta quebrada se toma el agua para el sistema de acueducto comunitario.

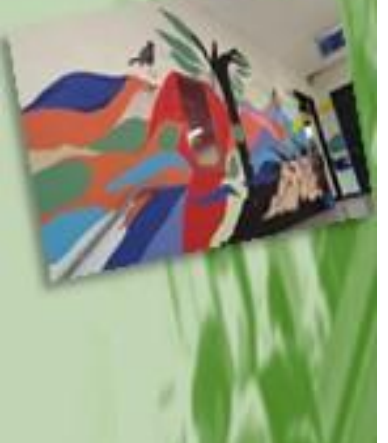

Así, dentro de las prácticas que ejecuta la población y afectan el ambiente y particularmente la Quebrada Morací sobresalen el arrojo de basuras, lo cual, revela una falta de apropiación y reconocimiento del mismo, hecho que pone en evidencia también el deterioro de las actitudes proambientales en la comunidad. Entendiendo antes, que una actitud ambiental refiere de acuerdo con Taylord y Todd (1995) citado por Álvarez \& Vega (2009, pág. 247) a "un determinante directo de la predisposición hacia acciones a favor del medio" donde dichas actitudes son muestra del actuar y el comportamiento de un sujeto frente a su ambiente. En esta medida, contribuir al potenciamiento de las actitudes en pro del ambiente, es un eje necesario para desarrollar y fortalecer indirectamente la relación estudiante-ambiente, y por tanto generar un sentido de pertenencia por el mismo, dado que, es pertinente iniciar un proceso de transformación con los estudiantes sobre aquellas acciones que afectan la estabilidad del ambiente.

Ahora bien, cabe señalar que las diversas expresiones han ampliado y complejizado el proceso de enseñanza aprendizaje de las diversas áreas de la educación escolar, en concordancia con esto, según Ruiz (2008) apoyado por Rojas (2013) el arte posibilita el desarrollo de habilidades motoras, cognitivas y creativas; que a su vez, permiten fortalecer procesos de sensibilización frente a su realidad, reconocer la incidencia de sus acciones, plasmando y representando sus conocimientos y experiencias poniendo a prueba su imaginación. No obstante, durante el proceso de contextualización e indagación con los estudiantes se halló que ésta es un área que se ha trabajado muy poco en la institución, dado que se dispone una hora a la semana para que los estudiantes construyan sus experiencias creativas, el desarrollo de sus habilidades y consecuentemente enriquezcan su formación integral, situación que permite comprender la importancia de integrar el arte con las ciencias para la enseñanza de la Educación Ambiental.

En este sentido, el planteamiento problema de la presente investigación surge teniendo en cuenta, las problemáticas mencionadas anteriormente. Así mismo, con este trabajo se pretende señalar la importancia del arte 
Bio-grafía. Escritos sobre la Biología y su Enseñanza. ISSN 2027-1034

Número Extraordinario. p.p. 86-99

Memorias del Primer encuentro ambiental Universidad, ambiente y sustentabilidad: experiencias y prácticas.

como área transversal que facilita y dinamiza el proceso de enseñanza aprendizaje de la Educación Ambiental en los estudiantes, permitiendo acercarse a la realidad de los mismos, a partir de la aplicación de diferentes expresiones artísticas como medio para fortalecer las actitudes ambientales de los estudiantes frente la Quebrada Morací.

\section{OBJETIVO}

Fortalecer las actitudes ambientales de los estudiantes frente a la Quebrada Morací a través de una estrategia pedagógica que incluya las expresiones artísticas.

\section{MARCO TEÓRICO}

Para el desarrollo de este apartado, se establecen tres categorías que aportan un mejor manejo de lo conceptual y además contribuyen a fundamentar mejor el trabajo investigativo, así se manejan:

\section{Expresiones artísticas una forma de interactuar con el mundo}

Las diferentes expresiones artísticas permiten poner a prueba la imaginación de los estudiantes para que plasmen sus ideas, experiencia y concepciones del mundo que posea. En este sentido se retoma a (Ruiz, 2008 pp. 5) quién se refiere a las expresiones artísticas como "el proceso psicopedagógico que estimula el libre desarrollo de la creatividad, fortalecimiento de relaciones grupales, la autoestima y el auto-concepto por medio de la aceptación y reconocimiento que se generan en el transcurso del proceso orientado como estrategia terapéutica $y$ educativa".

Es por ello que se puede encontrar una estrecha relación entre las expresiones artísticas y lo educativo, ya que entre estos se logra una contribución y transformación de la enseñanza aprendizaje de diversos contenidos educativos, por tanto, se puede encontrar que para (Rojas, 2013 pp. 43) la expresión artística, "es el conjunto de acciones y creaciones humanas que manifiestan sentimientos, emociones y belleza a través representaciones del arte, como la pintura, la poesía, el cuento, el teatro, el dibujo y cualquier otra disciplina de las artes plásticas, visuales, corporales y escénicas". En este sentido la relación que convoca el arte y la dimensión ambiental se generan a partir de: ser una posibilidad para despertar los sentires de los estudiantes frente a las realidades y las 
Bio-grafía. Escritos sobre la Biología y su Enseñanza. ISSN 2027-1034

Número Extraordinario. p.p. 86-99

Memorias del Primer encuentro ambiental Universidad, ambiente y sustentabilidad: experiencias y prácticas.

problemáticas multidimensionales que se encuentran en el contexto. Así, el arte propicia la sensibilización de los sujetos hacia el entorno, permitiendo fortalecer indirectamente las actitudes ambientales, transformando de forma progresiva sus prácticas frente al ambiente y aportando de igual forma a la creatividad de cada uno de ellos para brindar propuestas que permitan minimizar las problemáticas ambientales.

\section{Actitudes ambientales un reflejo de las acciones personales y colectivas}

La llegada de la educación ambiental a la escuela, trajo consigo ejes que representan una base teórica esencial para su enseñanza-aprendizaje, además de posibilitar una transformación de la realidad social, aunque no sean muy nombrados; las actitudes ambientales caracterizan las acciones de los sujetos. Ello lleva a plantear a Taylord \& Todd (1995) citado por Álvarez \& Vega (2009, pp. 247) a "la actitud ambiental como un determinante directo de la predisposición hacia acciones a favor del medio" señalando que "las actitudes y la intención de actuar tienen una importante influencia sobre el comportamiento..." de igual forma (Castro 2001, pp. 18) citado por (Valdeiglesis \& Aguilar sf, pp. 6) menciona que la actitud ambiental es "aquella acción que realiza una persona ya sea de forma individual o en un escenario colectivo, a favor de la conservación de los recursos naturales y dirigida a obtener una mejor calidad del medio ambiente". Teniendo en cuenta las definiciones anteriormente expuestas, se reconoce entonces la actitud ambiental como una acción a favor del ambiente, lo cual permite visibilizar que dichas actitudes poseen un objetivo en pro del mejoramiento del mismo, estableciendo una razón de ser, enmarcada por una intencionalidad, que caracteriza al individuo, en su relación sujeto-ambiente.

\section{Las estrategias pedagógicas desde el arte}

Las estrategias se han convertido en un eje central para la educación, favoreciendo la enseñanza-aprendizaje de cualquier área, o tema tanto para maestros como para estudiantes, enriqueciendo así su profesión y formación ciudadana. No obstante, son las estrategias pedagógicas de acuerdo con (Roa \& Toro, 2014 pp. 4) las que posibilitan "la reflexión de las prácticas permitiendo el encuentro entre conocimientos y saberes no privilegiando ninguno, pero si a partir de objetos de interés..." lo cual posibilita "Ias relaciones entre conceptos, sujetos e instituciones". De tal manera que el desarrollo de una estrategia pedagógica que retoma las 
Bio-grafía. Escritos sobre la Biología y su Enseñanza. ISSN 2027-1034

Número Extraordinario. p.p. 86-99

Memorias del Primer encuentro ambiental Universidad, ambiente y sustentabilidad: experiencias y prácticas.

diversas expresiones artísticas, debe orientar su proceso reflexivo hacia el por qué y para qué se está realizando, teniendo en cuenta cómo el campo o la educación artística logra nutrir y complementar los propósitos de la mismo contribuyendo al fortalecimiento de actitudes ambientales en los estudiantes, frente a su ambiente, así como al desarrollo de su inteligencia emocional y habilidades artísticas.

\section{METODOLOGÍA}

La metodología que se utilizó, se basa en un enfoque cualitativo, que según Rodríguez \& colaboradores en (1996) "la investigación cualitativa estudia la realidad de su contexto natural", idea que apoya Marshall y Rossman (1999) citado por Vasilachis (2006) mencionando que "la investigación cualitativa es pragmática, interpretativa y está asentada en la experiencia de la persona", esto indica que este tipo de investigación estudia la realidad social de los sujetos, a través de la interpretación, teniendo en cuenta el contexto y las interrelaciones que se tejen en los fenómenos sociales y culturales, es por ello, que este enfoque ha permitido desarrollar la experiencia educativa a partir del acercamiento de la realidad social de los estudiantes del grado 602, posibilitando explorar, describir y analizar la relación e interacción entre los alumnos y el ambiente, en donde se identificaron las actitudes que ellos presentan frente a la Quebrada Morací y las expresiones artísticas a trabajar.

En ese sentido se utiliza como método la Investigación Acción, que de acuerdo con (Kemmis \& McTaggart, 1988 citados por Suárez, M. 2002) "es una investigación que pretende mejorar la educación cambiando prácticas y que nos permite aprender gracias al análisis reflexivo de las consecuencias que genera." dichas prácticas deben ser registradas y analizadas a partir de diferentes técnicas e instrumentos de recolección de datos.

\section{La investigación se está desarrollando en 4 fases:}

1. Fase de observación y contextualización: consistió en el reconocimiento del contexto, del colegio y de los estudiantes del grado 602 por parte de las maestras en formación en donde se evidenció las problemáticas ambientales de la zona, en especial el fuerte impacto antrópico reflejado en el deterioro ecológico de la Quebrada Morací.

2. Fase de indagación, consistió en identificar con los estudiantes las actitudes pros ambientales a fortalecer, así como las expresiones 
Bio-grafía. Escritos sobre la Biología y su Enseñanza. ISSN 2027-1034

Número Extraordinario. p.p. 86-99

Memorias del Primer encuentro ambiental Universidad, ambiente y sustentabilidad: experiencias y prácticas.

artísticas de mayor interés en ellos, para lo cual propusieron 5 actividades que se desarrollan en el apartado de los resultados.

3. Fase de planeación e implementación, es la etapa en la que se encuentra el trabajo, donde con los estudiantes se están planteando, desarrollando y socializando las diferentes actividades artísticas.

4. Fase de reflexión, se ha centrado en la socialización de la experiencia posterior al desarrollo de las actividades hasta ahora implementadas, también, busca ser una fase final de la investigación donde se lleve a cabo una reflexión de todo el proceso y desarrollo de la propuesta pedagógica.

\section{RESULTADOS}

Este apartado se centra en analizar los resultados preliminares de los primeros momentos de la investigación, puesto que este trabajo se encuentra en desarrollo. Por tanto, a continuación, se presentan los resultados de la primera fase y los hallazgos parciales de la fase de indagación.

\section{Fase I Observación y contextualización}

Durante esta fase, se logró identificar las principales problemáticas presentes en el contexto, en la institución educativa y en los estudiantes, como se mencionó en un inicio existe un fuerte impacto antrópico sobre los diferentes ecosistemas que conforman el territorio reflejado en prácticas que afectan negativamente el ambiente, siendo común acciones como: el arrojo de basura dentro de la institución y en los diferentes escenarios naturales como lo es la Quebrada Morací, principal fuente hídrica del barrio y ecosistema con gran riqueza biológica y ecológica. Ello es evidencia de la importancia del fortalecimiento de actitudes pro ambientales que propicien un cambio social y ambiental dentro de la comunidad, donde se contribuya a generar un sentido de apropiación, respeto y cuidado por el ambiente.

Así mismo, dentro de esta fase fue posible encontrar que existe un marcado interés de los estudiantes por el desarrollo de las diversas expresiones artísticas, sin embargo, en el horario que maneja la institución, se establece una hora semanal para abordar la educación artística situación que incide en la formación integral de los estudiantes. Es por ello, que se decide tener en cuenta esta área como eje transversal para contribuir al fortalecimiento de actitudes pro ambientales, dado que posibilita que los estudiantes se expresen con mayor facilidad frente a las 
Bio-grafía. Escritos sobre la Biología y su Enseñanza. ISSN 2027-1034

Número Extraordinario. p.p. 86-99

Memorias del Primer encuentro ambiental Universidad, ambiente y sustentabilidad: experiencias y prácticas.

problemáticas y realidades del ambiente, es por tanto que esta investigación reconoce la importancia de impulsar la interdisciplinariedad entre áreas como la Educación Ambiental, biología y artes.

\section{Fase II Indagación}

Esta fase se encuentra constituida por 5 actividades que permitieron determinar las actitudes pros ambientales a fortalecer y las expresiones artísticas de mayor interés para los estudiantes

\section{Actividad I Encuesta Semi-estructurada}

La encuesta se constituyó por 7 preguntas que permiten dar cuenta de las actitudes que los estudiantes reconocen, las expresiones artísticas de mayor interés para abordar e identificar si consideran existe una relación entre las expresiones artísticas y el fortalecimiento de las actitudes pro ambientales.

Se encontró en primera instancia que los estudiantes relacionan las actitudes ambientales con acciones o comportamientos cotidianos, como lo expresa E11.P1 "No arrojar basura, no gasto tanta agua, recojo basura que encuentro en el piso o en el agua" y E26.P2 "Pues yo no voto a la calle muchos papeles, es más con mi tía y mi mamá cuando nos ven botando un papel nos lo hacen recoger y aparte nos hacen recoger 5 más", respuestas que demuestren el manejo de un conocimiento de actitud ambiental muy limitado, centrado en el actuar, olvidando que también están determinadas por sus valores, por la visión que se posee de ambiente y por las experiencias e interacciones que se han construido entre los sujetos y el ambiente.

Así mismo, se halló entre las actitudes pro ambientales que los estudiantes reconocen y han manejado intermitentemente a lo largo de su vida principalmente la responsabilidad, y el cuidado del ambiente, como lo menciona el E8. P1 "que soy responsable para cuidar el medio ambiente", y el E17.P1 "Pues cuando compro algo no los boto a las quebradas o a la calle si no a las canecas de basura".

Finalmente, se identifican las diferentes expresiones artísticas que los estudiantes conocen y les gustaría trabajar en las sesiones de clase, donde predominan el dibujo y la pintura, haciendo alusión a "Conozco el dibujo, con esto basta para expresar lo que sentimos" E4. P4. Por otro lado, el E9. P2 "Porque siento que estoy expresando mis sentimientos; porque casi todos dibujan; me expreso; todos los recuerdos; me siento 
Bio-grafía. Escritos sobre la Biología y su Enseñanza. ISSN 2027-1034

Número Extraordinario. p.p. 86-99

Memorias del Primer encuentro ambiental Universidad, ambiente y sustentabilidad: experiencias y prácticas.

feliz y más tranquila" en estas frases se puede ver que para ellos las expresiones artísticas cumplen un papel fundamental y es el poder expresar sus sentimientos.

\section{Actividad II Dibujo sobre la Quebrada Morací}

Esta actividad consistió en que los estudiantes debían realizar un dibujo de la Quebrada Morací, pero en esta se tuvieron inconvenientes, puesto que no todos los estudiantes no la conocen, aun siendo tan importante para la comunidad y cercana a ellos. Aun así, los dibujos que presentan hacen alusión a un ambiente contaminado, sin embargo, algunos plasmaron un ambiente sano y lleno de organismos que habitan el ecosistema.

\section{Actividad III Cartografía ambiental}

Con esta actividad se encontró que un considerable porcentaje de estudiantes no reconoce la Quebrada Morací, aun cuando viven en el Barrio (San Luis) o aledaño al mismo, hecho que determina la falta de reconocimiento de su contexto; aspecto que consecuentemente permite cuestionar el sentido de pertenencia y apropiación de los estudiantes por su territorio.

Por otro lado, se halló dentro de las cartografías, que la mayoría de los estudiantes identifican fácilmente la problemática ambiental más sobresaliente (el arrojo de basuras a las calles, y ecosistemas) dentro del contexto, sin embargo, no reconocen (algunos) los diferentes cuerpos de agua presentes en la zona, hecho que nuevamente pone en evidencia la pertinencia de abordar una educación ambiental contextualizada.

\section{Actividad IV Visión de ambiente}

Se realizó un dibujo en torno a la concepción de ambiente que manejaba cada estudiante, donde tuvieron la posibilidad de agregar a la ilustración una descripción de lo que significaba para ellos este término. Para el análisis de los dibujos y el texto anexo, se retomaron las diversas corrientes ambientales que propone Sauvé, (2004) lo cual posibilito establecer cómo están comprendiendo los estudiantes el ambiente desde dichas corrientes.

En este sentido, se encontró que el $27 \%$ de los estudiantes comprenden el ambiente desde la visión conservacionista o recursista, dado que lo consideran como los diversos escenarios naturales o ecosistemas 
Bio-grafía. Escritos sobre la Biología y su Enseñanza. ISSN 2027-1034

Número Extraordinario. p.p. 86-99

Memorias del Primer encuentro ambiental Universidad, ambiente y sustentabilidad: experiencias y prácticas.

constituidos por abundante biodiversidad lo cuales requieren ser protegidos porque son fuente primordial para la permanencia del hombre sobre el planeta, así el E3 explica "el ambiente para mí es muy importante porque sin oxígeno y sin agua no sobreviviríamos por eso toca cuidarlo y no desperdiciarlo" así mismo el E11 menciona "...la naturaleza es una de las razones de nuestra existencia y nuestra amiga”, de acuerdo con Sauvé (2004), el conservacionismo se centra en cuidar el ambiente, dado que es aquel que provee el alimento y satisface diversas necesidades a la sociedad, por tanto lo concibe como un recurso.

También se encontró que el 11,5\% de los estudiantes comprenden el ambiente desde la corriente humanista, dado que lo reconocen como el lugar donde se ubica tanto los escenarios naturales como los urbanos, una zona donde no sólo habitan animales y plantas sino también se encuentran e interaccionan los humanos, por ejemplo el E.8 menciona " $E l$ medio ambiente es un lugar o ecosistema donde habitan seres vivos como animales, plantas y humanos" así mismo el E26 dice "Es la unión de varias personas no solo donde hay plantas sino donde también hay urbanización", según Suave (2004), la corriente humanista permite identificar al sujeto dentro del ambiente, donde se reconoce su interacción directa y se concibe como parte del ambiente, es por tanto el resultado de la alianza entre lo natural con la construcción antrópica.

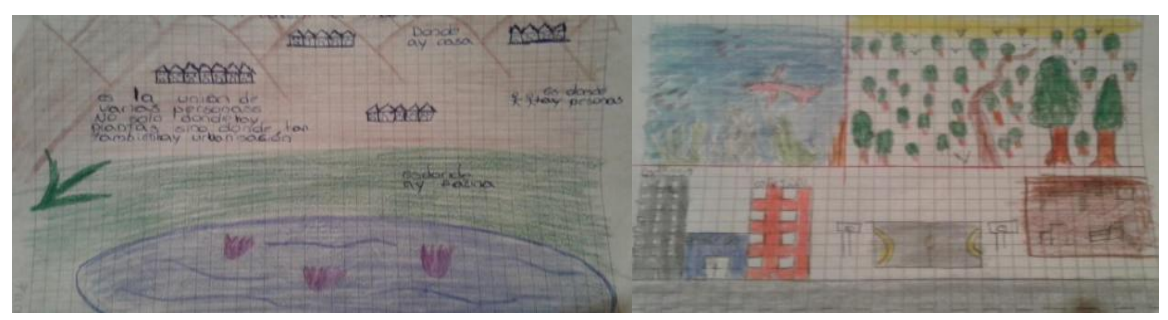

Imágenes 1. Ambiente desde la visión humanista (Dibujo Izquierda E8 y Dibujo derecha E26)

Por último, se encontró que el $57.7 \%$ de los estudiantes comprende el ambiente desde una visión naturalista, dado que considera y plasma su relación con la naturaleza, donde expresan aspectos experienciales así como su conocimiento en torno a la importancia de la naturaleza, y la riqueza de estos escenarios en cuanto a biodiversidad, por ejemplo a nivel experiencial el E18 menciona "Es lindo y en donde uno puede tener paz" y el E5 dice "El ambiente para mí es un medio de tranquilizaciòn con la naturaleza y es una parte muy linda la cual estamos arruinando" de igual forma el E4 estable a nivel de conocimiento disciplinar que "El medio ambiente está dividido en diferentes reinos, ecosistemas y sistemas 
Bio-grafía. Escritos sobre la Biología y su Enseñanza. ISSN 2027-1034

Número Extraordinario. p.p. 86-99

Memorias del Primer encuentro ambiental Universidad, ambiente y sustentabilidad: experiencias y prácticas.

respiratorios, que hacen a cada animal diferente... hay tanto animales en el agua, como en el cielo y en la tierra", estos son evidencia de la concepción de ambiente que poseen los estudiantes también desde su interacción permanente, dado seguramente la mayoría de ellos viven en una zona semiurbana, caracterizada por poseer diferentes ecosistemas.
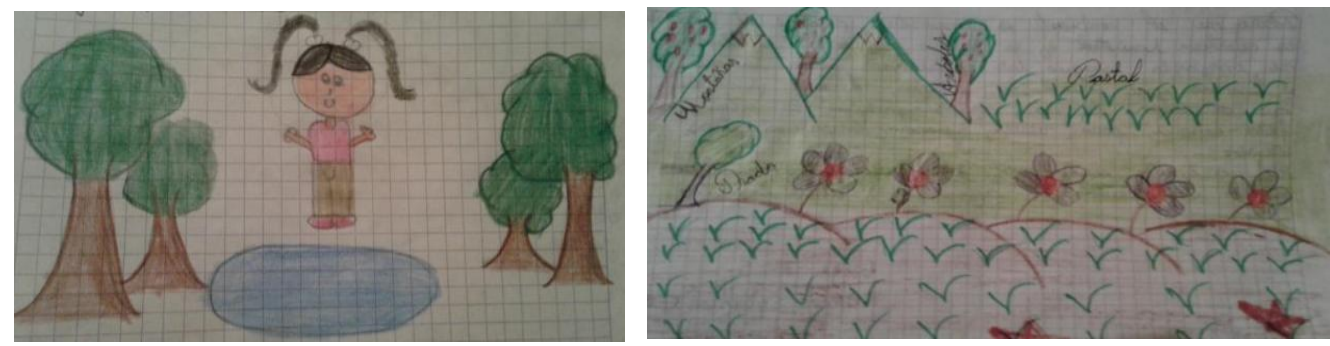

Imagen 2. Visión naturalista de ambiente. (Dibujo IzquierdaE13, Dibujo derecha E3)

A nivel general, otro de los resultados más distintivos es la concepción de ambiente desde una sola dimensión, la mayoría de los estudiantes relacionó ambiente directamente con la naturaleza o ecosistemas, muy pocos integraron a su definición y representación gráfica aspectos culturales, sociales, y políticos, que hacen al ambiente multidimensional y complejo. Así mismo, se identificó que un $88,5 \%$ de los jóvenes se consideran independientes del ambiente, como sujetos alejados que no hacen parte de la naturaleza y por tanto del ambiente.

\section{Actividad V Representación mímica}

Esta actividad, se dividió en dos momentos, el primero consistió en representar a través de la mímica algunas de las actitudes pro ambientales abordadas en la encuesta, o actitudes que ellos manejaban. Así, se decidió representar por grupos una actitud, dentro estas se eligieron: la responsabilidad, la concienciación, la conservación, el reconocimiento del contexto, la proposición y resolución y la igualdad ambiental (considerar al hombre y la ambiente como iguales), con el desarrollo de las representaciones, se encontró, que los estudiantes se ciernen a actuaciones que involucren el no arrojo de basura a las calles, y no abarcan otras problemáticas ambientales comunes en su contexto.

Posterior al ejercicio mímico, se llevó a cabo una socialización de la actividad, durante este momento los estudiantes por grupos determinaron las 3 actitudes pro ambientales que consideran necesario fortalecer a 
Bio-grafía. Escritos sobre la Biología y su Enseñanza. ISSN 2027-1034

Número Extraordinario. p.p. 86-99

Memorias del Primer encuentro ambiental Universidad, ambiente y sustentabilidad: experiencias y prácticas.

través del arte, así el 88\% de ellos consideró necesario iniciar el proceso con el reconocimiento del contexto, dado que es un actitud que permite conocer su ambiente, establecer la importancia de los ecosistemas acuáticos y particularmente de la Quebrada Morací, e identificar la biodiversidad presente en dicha zona, así el G2 mencionó "el reconocimiento del contexto porque debemos conocer el lugar 0 ecosistema donde vivimos" de igual forma el G5 expresó "decidimos trabajar reconocimiento del contexto porque vamos a poder reconocer los sitios donde hay más contaminación y aprender a cómo ayudar esos sitios". Así mismo, otro $88 \%$ de los niños coincidió en la pertinencia de abordar la proposición y resolución de problemáticas ambientales, como actitud que posibilita pensar en propuestas para dar posibles soluciones a las diferentes situaciones negativas, por ejemplo, el G6 menciona en "proponer ideas para hacer una campaña para mejorar el medio ambiente". Por último, un $50 \%$ de los estudiantes mencionó la responsabilidad como la última actitud a trabajar durante el proceso de fortalecimiento, dado que consideran necesario poseer una actitud responsable frente a su territorio, donde exista un cuidado y respeto por el mismo, de esto modo el G6 menciona "El ambiente es parte de nosotros y si no lo cuidamos se destrozará. Ser responsables y cuidar el ambiente".

De acuerdo con los resultados obtenidos durante la actividad, se decidió con los estudiantes que las actitudes ambientales que se trabajarían con el fin de contribuir a su fortalecimiento por medio de las artes serian: el reconocimiento del ambiente, la proposición y resolución de problemáticas ambientales y la responsabilidad, dado que son actitudes que permiten indirectamente generar un sentido de pertenencia y apropiación por su ambiente, y frente a los ecosistemas que conforman dicha zona.

\section{CONCLUSIONES}

Las actividades que hasta el momento se han desarrollado, permiten evidenciar que los estudiantes son conscientes del deterioro del ambiente a causa de las malas prácticas propias y que se generan en la comunidad, una de las problemáticas más evidente en el contexto es la contaminación de los ecosistemas con los que cuenta el barrio y principalmente el fuerte impacto que esto tiene frente a la quebrada Morací, reconociendo la importancia de mejorar esas prácticas en pro del ambiente. Durante este primer acercamiento con los estudiantes y sus concepciones sobre su ambiente, es notable que ellos ven el ambiente como un ecosistema o un espacio netamente natural, en donde muy 
Bio-grafía. Escritos sobre la Biología y su Enseñanza. ISSN 2027-1034

Número Extraordinario. p.p. 86-99

Memorias del Primer encuentro ambiental Universidad, ambiente y sustentabilidad: experiencias y prácticas.

pocos se incluyen o incluyen otros factores biofísicos que hacen parte de este, como lo es la urbanización. Por otro lado, se logra evidenciar que los estudiantes son conscientes y conocen las diferentes problemáticas que están presentes en su contexto, pero son ellos mismos los que a través de sus escritos dejan ver que quieren un cambio de prácticas que sean en pro del ambiente, para cuidar los diferentes ecosistemas que conforman el contexto.

El desarrollo de estas primeras actividades permite reflexionar sobre el quehacer docente y sobre el mejoramiento continuo de estas actividades, para que los estudiantes tengan un aprendizaje significativo, puesto que se ve la relevancia de fortalecer en los alumnos las actitudes pro ambientales para generar un cambio social y ambiental en la comunidad escolar.

\section{BIBLIOGRAFÍA}

Álvarez, P y Vega, P. (2009). Actitudes ambientales y conductas sostenibles. Implicaciones para la Educación Ambiental. Vol. 12, número 2, pop 242-260, Revista de Psicodidáctica. Universidad del País Vasco. España.

Alvarado, L \& García M. (2008). Características más relevantes del paradigma socio-crítico: su aplicación en investigaciones de educación ambiental y de enseñanza de las ciencias realizadas en el Doctorado de educación en el Instituto pedagógico de Caracas. Sapiens, Revista Universitaria de Investigación. Caracas, Venezuela.

Cerda, H. (2002). Los elementos de la investigación: cómo reconocerlos, diseñarlos y construirlos. Editorial el Buho. Bogotá -Colombia

Hernandez, M. (19 de Julio de 2013). Biodiversidad y Conservación.

Obtenido de Biodiversidad y Conservación:

http://biodiversidadyconservacion.blogspot.com.co/2013/07/cerros-al-

oriente-de-bogota.html

Rodríguez, G. et.al. (1996). Metodología de la investigación cualitativa.

Ediciones Aljibe. Granada- España. 
Bio-grafía. Escritos sobre la Biología y su Enseñanza. ISSN 2027-1034

Número Extraordinario. p.p. 86-99

Memorias del Primer encuentro ambiental Universidad, ambiente y sustentabilidad: experiencias y prácticas.

Suárez, M. (2002). Una reflexión sobre algunas cuestiones relacionadas con la investigación-acción colaboradora en educación. Revista electrónica de enseñanza de las ciencias, Vol. 1. N 1 pp 40-56. Universidad de Vigo. España.

Vasilaches, I. (2006). Estrategias de investigación cualitativa. Gedisa editorial. Barcelona, España. 